\title{
FACTORES DE DESERCIÓN DE LOS ESTUDIANTES EN LA FACULTAD DE ENFERMERÍA DE LA UNIVERSIDAD DE CIENCIAS APLICADAS Y AMBIENTALES U.D.C.A, DURANTE EL PERIODO: 2009-2010-I 2011
}

\author{
STUDENT DROPOUT FACTORS OF THE SCHOOL \\ OF NURSING, UNIVERSITY OF APPLIED AND \\ ENVIRONMENTAL SCIENCES U.D.C.A, PERIOD: \\ 2009-2010-I 2011
}

\author{
Blanca N. Piratoba H. ${ }^{1}$, Ovidio O. Barbosa Ch. ${ }^{2}$
}

\begin{abstract}
${ }^{1}$ Enfermera, Especialista en Administración de Servicios de Salud, Magister en Enfermería con énfasis en Cuidado al Paciente crónico. Profesora Facultad de Enfermería. Universidad de Ciencias Aplicadas y Ambientales U.D.C.A, Calle 222 No 55- 37, Bogotá D.C., Colombia, blancapiratoba@hotmail.com; ${ }^{2}$ Enfermero, Joven investigador U.D.C.A, Universidad de Ciencias Aplicadas y Ambientales U.D.C.A, Calle 222 No 55- 37, Bogotá D.C., Colombia, ovidiobarbosa_2005@hotmail.com.
\end{abstract}

Rev. U.D.C.A Act. \& Div. Cient. 16(2): 553-562, Julio-Diciembre, 2013

\begin{abstract}
RESUMEN
El objetivo de la investigación fue identificar los factores económicos, académicos, socio-culturales y otros, asociados a la deserción de los estudiantes en la Facultad de Enfermería, de la Universidad de Ciencias Aplicadas y Ambientales U.D.C.A, en cada una de las de las cohortes, de los años 2009, 2010 y primer semestre de 2011. Bajo un estudio descriptivo con abordaje cuantitativo retrospectivo, se obtuvieron los datos del sistema de la universidad, a través Departamento de Planeación, de Sistema de Admisiones y Registro Académico y de la Facultad de Enfermería. En las cinco cohortes, se identificaron 144 desertores, de los cuales, 59 corresponden a la muestra. Para la recolección de la información, se utilizó un instrumento diseñado por el Programa de acompañamiento y adaptado por los investigadores, con 33 ítems, que incluían variables de tipo individual, personal, socio-económicas y académicas; se aplicó previo consentimiento informado. Los resultados mostraron los primeros semestres como el momento en el que se presenta el mayor número de desertores y en las edades de 16 a 20 años, con un $47 \%$. Las principales causas asociadas a la deserción fueron los factores económicos, con un 28,8\%, seguido de los factores académicos, con un $22 \%$. La unión de factores económicos y académicos es la tercera causa de deserción, con un 15,3\%, mientras que la orientación vocacional, se encuentra en cuarto lugar, con un $11,9 \%$. En menor porcen-
\end{abstract}

taje, se detectaron otros factores, como el no adaptarse al ambiente universitario, condiciones de salud, embarazo y el cambio de lugar de residencia.

Palabras clave: Abandono, factores de riesgo, educación superior universitarios.

\section{SUMMARY}

The objective of the research was to identify the economic, academic, cultural and other factors, associated to student dropout in the faculty of nursing at the University of Applied Sciences and Environmental Sciences in each of the cohort of 2009, 2010 and 2011, I. Corresponds to a descriptive study with retrospective quantitative approach, supported by units of the university and the Department of Planning, Admissions and Registration System Academic, and nursing faculty. In the five cohorts 144 deserters were identified, 59 corresponded to the sample. To collect the information an instrument designed by the accompanying program and adapted by the researcher with 33 items, including variables of an individual, personal, socioeconomic and academic type was used. Informed consent was implemented. The results showed the first semester as the moment of the highest number of dropouts, being higher in females between the ages of 16 to 20 years with a $47 \%$. The main causes of dropout were economic factors with $28.8 \%$, followed by academic ones with $22 \%$, the union 
of economic and academic factors are the third leading cause of attrition with $15.3 \%$, while vocational guidance is in fourth place with $11.9 \%$; lower percentage of factors such as failure to adapt to the universities environment, health conditions such as illness or pregnancy, change of residence to another city, were detected.

Key words: Dropouts, risk factors, higher education.

\section{INTRODUCCIÓN}

La deserción estudiantil es uno de los problemas que se presenta en la mayoría de las Instituciones de Educación Superior de Latinoamérica (Díaz Peralta, 2008). El estudio de esta deserción es extremadamente complejo, ya que implica no solamente una variedad de perspectivas, sino una gama de diferentes tipos de abandono. Adicionalmente, se afirma que ninguna definición puede captar en su totalidad la complejidad de este fenómeno, quedando en manos de los investigadores la elección de la definición que mejor se ajuste a sus objetivos y al problema a investigar (Tinto, 1989).

Existe consenso en definir la deserción como un abandono voluntario, que puede ser explicado por diferentes categorías de variables: socio-económicas, individuales, institucionales y académicas (Díaz Peralta, 2008). Así que este proceso de abandono, voluntario o forzoso de la carrera en la que se matricula un estudiante, por la influencia positiva o negativa de circunstancias internas o externas a él o ella (UNESCO/ IESALC, 2006) es una problemática que ha tomado especial relevancia, en las últimas décadas (González, 2005). Según estadísticas, en el caso de las Instituciones de Educación Superior Colombianas, la deserción está cerca del 50\%, es decir, la mitad de los estudiantes quienes inician una formación profesional no la finaliza (Bravo Castillo \& Mejía Giraldo, 2010).

Diferentes autores intentan explicar las causas de la deserción, mediante modelos que describen cómo se produce la decisión de desertar; los enfoques psicológicos de la retención estudiantil emplean variables de tipo individual, que se centran en los rasgos de la personalidad que diferencian a los alumnos que completan sus estudios de aquellos quienes no lo logran (Himmel, 2002). El primer modelo que aparece en la literatura es el de Fishbein \& Ajzen (1975), quienes señalan que desertar o permanecer está directamente influenciado por las conductas previas, las actitudes acerca de la deserción y las normas subjetivas acerca de estas acciones.

Los modelos sociológicos enfatizan en la influencia en la deserción de factores externos al individuo, adicionales a los psicológicos. Spady (1970), se basó en el modelo suicida de Durkheim (1951), indicando que la deserción es el resultado de la falta de integración de los estudiantes en el entorno de la educación superior; aduce, que el medio familiar es una de las muchas fuentes que expone a los estudiantes a influencias, expectativas y demandas, las que, a su vez, afectan su nivel de integración social en la universidad; la congruencia normativa actúa directamente sobre el rendimiento académico, el desarrollo intelectual, el apoyo de pares y la integración social, situaciones que generan una alta probabilidad que el estudiante decida abandonar sus estudios. Los modelos organizacionales hacen referencia a las características de la deserción en las instituciones, en cuanto a los servicios que estas ofrecen a los estudiantes, que ingresan a ella (Himmel, 2002), cobrando especial relevancia la calidad de la docencia (Braxton et al. 1997) y las experiencias de los estudiantes en el aula (Himmel, 2002). Los factores sociales, económicos o familiares influyen en la deserción universitaria, siendo más representativos los económicos (Rojas Betancur E González, 2008).

El Sistema para la Prevención de la Deserción de la Educación Superior -SPADIES- muestra, que en promedio, la tasa de deserción acumulada entre 1998 y el 2005, en la U.D.C.A fue de 50,16\%, lo que significa, que por cada 100 estudiantes que ingresaron en la cohorte, 50 se retiraron, antes de obtener su grado (MEN, 2008).

La alta deserción en la Educación Superior (ES) está asociado con calificaciones bajas al ingreso, los estudiantes con padres que no han tenido educación universitaria o con estatus socio-económico bajos (Jeffreys, 2007).

La deserción en la formación de Enfermeras y Enfermeros es de las principales preocupaciones, a nivel mundial, para los gobiernos, para las Instituciones de educación superior y para la profesión (Pryjmachuk et al. 2009). En la enfermería y la partería, el debate se centra en torno a la deserción, donde se reporta, como posibles razones, las personales en lugar de los factores sociales, que pueden influir en su capacidad para permanecer en el programa (Cameron et al. 2011). Aspectos académicos, ubicación, finanzas y asuntos personales fueron citados por los estudiantes, como factores que les hizo pensar en abandonar el programa de Enfermería (Bowden, 2008).

Las razones para el abandono son multifactoriales, que corresponden a problemas identificados, como personales, carga financiera, angustia emocional, dificultad de afrontamiento, dificultades académicas, problemas de ubicación laboral y toma de decisión equivocada con la carrera, (Glogowska et al. 2007).

Este estudio buscaba identificar los factores que determinaron la deserción de los estudiantes del Programa de Enfer- 
mería de la Universidad de Ciencias Aplicadas y Ambientales U.D.C.A.

\section{MATERIALES Y MÉTODOS}

Es un estudio descriptivo con abordaje cuantitativo retrospectivo y los datos se recolectaron del Sistema de Información de la Universidad, a través del Departamento de Planeación, del Sistema de Admisiones y Registro Académico y de la Facultad de Enfermería. La población estuvo conformada por 144 estudiantes desertores del programa de cinco cohortes: 364 estudiantes, entre el 2009, 2010 y primer semestre de 2011. En cada cohorte, se tuvo en cuenta el número total de estudiantes matriculados, haciendo el seguimiento durante los siguientes periodos académicos, hasta el primer semestre de 2011, para identificar el número de estudiantes que permanecían y determinar el número total de desertores, por cohorte.

Para la recolección de la información, se utilizó el Instrumento diseñado por el Programa de Acompañamiento de la Universidad de Ciencias Aplicadas y Ambientales U.D.C.A, con 33 ítems; las variables consideradas fueron de tipo individual y personal, que incluían: sexo, edad de ingreso, ciudad, departamento de origen, tipo de colegio, orientación vocacional, periodo académico de ingreso y retiro; variables socio-económicas: estado civil, personas quienes financiaron estudios y su actividad económica, forma de pago de la matrícula, educación del padre y la madre, becas y ayudas de manutención, si trabajó durante el periodo de estudios; variables académicas, como: causas que influyeron en la toma de decisión de abandonar el programa, dificultades de permanencia, búsqueda de apoyo antes de abandonar el programa, estudios posteriores a abandonar el programa, cuántos semestres cursó antes de desertar y actividad a la que se dedica en la actualidad. El instrumento fue adaptado por los investigadores, previa autorización de los autores, para su utilización y modificación. El estudio recibió el aval del comité de ética Institucional, como requisito en convocatoria interna; prevalece en el estudio, el respeto a la dignidad y la protección de los derechos expresado en el consentimiento informado y escrito del sujeto de investigación.

Se empleó muestreo no probabilístico de tipo intencional, con una muestra de 59 participantes. Dentro de las características socio-demográficas y culturales de los desertores de la Facultad de Enfermería de la U.D.C.A para $(n=59)$ según género, el $86 \%$ fueron mujeres y $14 \%$ hombres.

Para la identificación de los desertores, se inició seguimiento a cada grupo de estudiantes matriculados en cada cohorte, contrastando los datos con la información reportada por el Sistema de Admisiones y Registro Académico -SARA-, Departamento de Planeación y la Facultad de Enfermería.
Para la obtención de la información, se solicitaron los permisos institucionales a los departamentos responsables. Se emplearon diferentes medios para ubicar y aplicar el instrumento a los estudiantes que desertaron: el 38,9\%, a través de visitas domiciliarias; $22 \%$, por redes sociales; $20 \%$, por intermedio de compañeros y, el 18,6\%, telefónicamente.

Se creó la base de datos en Excel, se tomaron las diferentes tipo de variables del instrumento, a nivel individual, personal socio-económicas y académicas, se generaron categorías y se tabularon en la base; posteriormente, se transportó al programa SPSS versión 19.0 y se hizo un análisis con estadística descriptiva.

\section{RESULTADOS Y DISCUSIÓN}

La tabla 1 muestra las causas de deserción agrupadas por semejanzas. Una característica particular de los programas de enfermería del país es que las mujeres son mayoría dentro de la profesión (Sanabria, 2002). En diferentes instituciones de educación predomina la deserción del género femenino para el área ciencias de la salud (Yepes et al. 2007; Carvajal Olaya et al. 2006; Mateus Rodríguez et al. 2011).

Nigenda et al. (2006), en estudio realizado en México, muestra que la Licenciatura en Enfermería continúa siendo una ocupación altamente feminizada, a pesar del incremento de la presencia de hombres. Aunque el número de hombres en la enfermería están aumentando, permanecen minoritariamente representados (6\%) (Jeffreys, 2010).

La tabla 2 resume los factores relevantes, encontrados en este estudio, relacionados con la deserción de estudiante de Enfermería de la U.D.C.A.

Las edades que predominan en el programa de Enfermería de la U.D.C.A son de 16 a 20 años, datos similares con otras Universidades, como la del Cauca, en Popayán y la Tecnológica de Pereira (Chávez et al. 2002; Carvajal Olaya et al. 2006; Restrepo, 2010). La edad del estudiante determina el nivel de madurez y la claridad frente a lo que se quiere estudiar. La mayoría de estudiantes son adolescentes, etapa, en la cual, no se tiene orientación sobre la profesión que se quiere estudiar. Este comportamiento no se da en los estudiantes de Enfermería maduros o mayores, quienes tienen tendencia a terminar más que los jóvenes (Pryjmachuk et al. 2009).

En cuanto al tipo de colegio donde terminaron los estudios de básica segundaria, el 52,5\% proviene de colegios privados, calendario A; un $45,8 \%$, de colegios públicos y solamente un $1,7 \%$, de instituciones privadas, calendario B. Según el Sistema para la Prevención de la Deserción en las Instituciones de Educación Superior (MEN, 2008), el 59\% de los estudiantes 
Tabla 1. Causas de deserción comparativa de diferentes Universidades.

\begin{tabular}{|c|c|c|c|c|c|c|c|c|}
\hline \multirow[b]{2}{*}{ INSTITUCIÓN } & \multicolumn{8}{|c|}{ VARIABLE } \\
\hline & $\begin{array}{c}\text { Género } \\
\text { (femenino) }\end{array}$ & Edad & $\begin{array}{l}\text { Tipo de } \\
\text { colegio } \\
\text { (público) }\end{array}$ & $\begin{array}{l}\text { Estado } \\
\text { civil } \\
\text { (soltero) }\end{array}$ & $\begin{array}{l}\text { Factores } \\
\text { económicos }\end{array}$ & $\begin{array}{c}\text { Factores } \\
\text { académicos }\end{array}$ & $\begin{array}{l}\text { Periodos } \\
\text { académicos } \\
\text { cursados por } \\
\text { desertores }\end{array}$ & $\begin{array}{c}\text { Condición } \\
\text { académica } \\
\text { después de } \\
\text { desertar }\end{array}$ \\
\hline $\begin{array}{c}\text { UNIVERSIDAD } \\
\text { DE CIENCIAS } \\
\text { APLICADAS Y } \\
\text { AMBIENTALES } \\
\text { U.D.C.A }\end{array}$ & $86 \%$ & $\begin{array}{c}16-20 \\
\text { años } \\
49 \%\end{array}$ & $45,8 \%$ & $76 \%$ & $28,8 \%$ & $22 \%$ & $\begin{array}{c}50,9 \% \\
1 \text { periodo } \\
\text { académico }\end{array}$ & $\begin{array}{c}\quad 62,7 \\
\text { Actividades } \\
\text { laborales }\end{array}$ \\
\hline $\begin{array}{l}\text { UNIVERSIDAD } \\
\text { DEL CAUCA } \\
n=202\end{array}$ & $56,4 \%$ & $\begin{array}{l}15-19 \\
\text { años } \\
75,7 \%\end{array}$ & $65,8 \%$ & $95,5 \%$ & $66,7 \%$ & 33,3 & $\begin{array}{c}64,3 \% \\
3 \text { periodos } \\
\text { académicos }\end{array}$ & \\
\hline $\begin{array}{l}\text { UNIVERSIDAD } \\
\text { ANTIOQUIA } \\
n=50\end{array}$ & $59 \%$ & $\begin{array}{c}20-23 \\
\text { años } \\
68,9 \%\end{array}$ & $41,2 \%$ & $76,5 \%$ & $\begin{array}{c}\text { Cuarto } \\
\text { lugar } \\
\text { Motivos } \\
\text { económicos }\end{array}$ & $\begin{array}{l}41 \% \\
\text { Buen } \\
\text { rendimiento }\end{array}$ & $\begin{array}{c}29,4 \% \\
4 \text { periodos } \\
\text { académicos }\end{array}$ & $\begin{array}{c}77,8 \\
\text { Deseo de } \\
\text { trabajo }\end{array}$ \\
\hline $\begin{array}{l}\text { UNIVERSIDAD } \\
\text { LOS LLANOS } \\
n \mathrm{n}=500\end{array}$ & $41 \%$ & $\begin{array}{c}22-24 \\
\text { años } \\
44 \%\end{array}$ & $62 \%$ & & $26 \%$ & $47,8 \%$ & $\begin{array}{l}1-3 \text { periodos } \\
\text { académicos }\end{array}$ & $\begin{array}{c}51,2 \% \\
\text { Estudiando }\end{array}$ \\
\hline $\begin{array}{l}\text { UNIVERSIDAD } \\
\text { TECNOLÓGICA } \\
\text { PEREIRA } \\
n=58\end{array}$ & $55 \%$ & $\begin{array}{c}18-19 \\
\text { años } \\
39,7\end{array}$ & $66 \%$ & $93 \%$ & $20,7 \%$ & $8,6 \%$ & $\begin{array}{c}81 \% \\
1-2 \text { periodos } \\
\text { académicos }\end{array}$ & \\
\hline $\begin{array}{c}\text { UNIVERSIDAD } \\
\text { LIBRE PEREIRA } \\
n=36\end{array}$ & $83,3 \%$ & $\begin{array}{l}\geq 20 \\
\text { años } \\
65,7 \%\end{array}$ & $72 \%$ & $86 \%$ & $48,2 \%$ & & $\begin{array}{c}27,8 \% \\
1-2 \text { periodos } \\
\text { académicos }\end{array}$ & \\
\hline $\begin{array}{c}\text { UNIVERSIDAD } \\
\text { SAN } \\
\text { BUENAVENTURA } \\
n=20\end{array}$ & $65 \%$ & $16-32$ & & $90 \%$ & $35 \%$ & $65 \%$ & $\begin{array}{c}75 \% \\
\text { 1-3 periodos } \\
\text { académicos }\end{array}$ & \\
\hline
\end{tabular}


Tabla 2. Factores relevantes relacionados con la deserción universitaria U.D.C.A. $n=59$.

\begin{tabular}{|c|c|c|}
\hline Variable & $\mathrm{n}$ & $\%$ \\
\hline \multicolumn{3}{|l|}{ Género } \\
\hline Hombre & 8 & 14 \\
\hline Mujer & 51 & 86 \\
\hline \multicolumn{3}{|l|}{ Edad del desertor } \\
\hline 16-20 años & 29 & 49 \\
\hline $21-25$ años & 22 & 37 \\
\hline Mayor a 26 años & 8 & 13,6 \\
\hline \multicolumn{3}{|l|}{ Colegio de donde provienen } \\
\hline Privado calendario A & 31 & 52,5 \\
\hline Públicos & 27 & 45,8 \\
\hline Privado calendario B & 1 & 1,7 \\
\hline \multicolumn{3}{|l|}{ Estado civil } \\
\hline Solteros & 45 & 76 \\
\hline Unión Libre & 10 & 16,9 \\
\hline Casados & 4 & 6,8 \\
\hline \multicolumn{3}{|l|}{ Factores por los que desertaron } \\
\hline Económico & 17 & 28,8 \\
\hline Académico & 13 & 22 \\
\hline La unión de dos factores (económicos y académicos) & 9 & 15,3 \\
\hline Falta de orientación vocacional & 7 & 11.9 \\
\hline \multicolumn{3}{|l|}{ Dependencia económica del desertor } \\
\hline De la madre & 23 & 39 \\
\hline De la madre y el padre & 15 & 25 \\
\hline Recursos propios & 13 & 22 \\
\hline Solamente del padre & 3 & 5 \\
\hline \multicolumn{3}{|c|}{ Actividad económica de las personas que financiaban estudios de los desertores } \\
\hline Empleados & 42 & 71 \\
\hline Independientes & 13 & 22 \\
\hline Pensionados & 4 & 6,8 \\
\hline \multicolumn{3}{|l|}{ Semestres cursados } \\
\hline Un semestre & 30 & 50,9 \\
\hline Dos semestres & 14 & 22,8 \\
\hline Tres semestres & 10 & 17,5 \\
\hline Cuatro semestres & 1 & 1,7 \\
\hline
\end{tabular}


desertores provienen de instituciones de educación privadas y, el $41 \%$, de públicas. En Universidades con programas de Enfermería, como la Universidad Libre de Pereira, el 58,8\% de los estudiantes procedían de instituciones privadas (Restrepo, 2010); para la Universidad del Cauca, el 65,8\% de colegios públicos (Chávez et al. 2002) y para la Universidad Tecnológica de Pereira, el 66\% de los estudiantes de Ciencias de la Salud, de colegio público (Carvajal Olaya et al. 2006).

Los estudiantes provienen del sector privado y público para las diferentes Universidades. El nivel socio-económico y el capital cultural de las familias son los principales factores que explican las diferencias de rendimiento, tanto entre los estudiantes como entre los establecimientos. Los alumnos con dificultades financieras, con menor capital social y quizá con una educación de menor calidad, ingresan, finalmente, a planteles de baja selectividad y tienen un riesgo más alto de desertar del sistema (Saldaña E Barriga, 2010). El ingreso a la Universidad está mediado por una prueba de selección; los planteles de calidad siempre tienen mayor demanda, por lo que serán los que capten a los mejores alumnos, convirtiéndose, potencialmente, en un factor de riesgo para la deserción (Saldaña E Barriga, 2010).

La soltería, estado civil predominante en los desertores de la U.D.C.A, corresponde con otras Universidades, como la del Cauca, con el 95,5\% (Chávez et al. 2002); 93\%, en la Universidad Tecnológica de Pereira (Carvajal Olaya et al. 2006); $86 \%$, en la Universidad Libre de Pereira (Restrepo, 2010) y en la Universidad de Antioquia, 76,5\% (Yepes et al. 2007). El estado civil es una variable que se relaciona con la edad, teniendo en cuenta que la mayoría son jóvenes y en su proyecto de vida no está contemplado el casarse; la mayoría de los estudiantes quienes ingresaron solteros mantuvieron este estado al desertar.

En cuanto a los factores económicos, el 39\% de los desertores dependían económicamente de la madre; el 25\%, del padre y la madre; es importante resaltar que el $22 \%$ de los desertores asumió los costos de su formación con recursos propios; el 5\% dependía únicamente del padre; el 5\% dependía de recursos subsidiados por la madre y el 3,4\%, se apoyaba económicamente de otro familiar. Los estudios revisados muestran que esta situación cambia dependiendo de la institución, pero se mantiene el patrón de dependencia de los padres. Las condiciones económicas de la familia son determinantes y facilitan a los estudiantes permanecer en un programa. Si se afecta económicamente la familia, se verá afectado el estudiante para continuar vinculado a la Universidad, si no cuenta con recursos económicos disponibles, para los gastos que exige la carrera.

La actividad económica de las personas quienes financiaban a los estudiantes de la U.D.C.A, el $71 \%$ eran empleados; el
$22 \%$, independientes y el 6,8\%, pensionados, situación que determina la manera como pagan el semestre: el 62,7\%, lo hace en efectivo; el $23,7 \%$, a través de crédito bancario; el $8,5 \%$, por crédito ICETEX y el $5 \%$, emplean la modalidad de cheques.

El nivel socio-económico - tener un empleo o ingresos fijos por diferentes actividades-, permite a las familias apoyar a los estudiantes para realizar los pagos requeridos y mantener la vinculación del estudiante en la Universidad. Es evidente que el acceso a créditos es mínimo. La garantía del pago, se relaciona con la vinculación laboral y el ingreso mensual de las personas, quienes asumen la responsabilidad económica del estudiante. Las Instituciones de Educación Superior deben considerar que los estudiantes requieren de mayor apoyo económico y deben ampliar la oferta y el acceso a créditos educativos, para evitar el abandono.

El nivel educativo de las madres de los desertores de enfermería es superior a la de los padres, pues el 33,9\% de las mujeres poseían un nivel de formación universitaria, frente a un $20,7 \%$, en los hombres; sin embargo, los padres presentan un mayor nivel de formación en la básica secundaria, con un $46,6 \%$, frente a un 30,5\%, para las madres. Los datos para la U.D.C.A y los datos presentados con el programa de Enfermería en la Universidad Libre de Pereira muestran diferencias significativas, donde el $25 \%$ las madres de los estudiantes desertores poseían formación universitaria completa, frente a un 8,6\%, en los hombres (Restrepo, 2010).

El nivel de educación de los padres es una variable que influye en los factores que determinan la deserción, especialmente, el de la madre, en razón que a mayor nivel de educación de los padres, se presenta una menor deserción. Según los datos presentados por el Sistema para la Prevención de la Deserción en las Instituciones de Educación Superior-SPADIES- describen que los estudiantes desertores pertenecen, en mayor porcentaje, a familias que han alcanzado un nivel de formación de básica primaria en un 30,7\% y, en menor porcentaje, a quienes han alcanzado un nivel de superior completo o postgrado, con un $20 \%$ (MEN, 2009).

Con relación al interrogante si los estudiantes desertores trabajaban o no, el estudio mostró que el 23,7\%, lo hacían durante la época de estudios, con el fin de obtener de recursos de sostenimiento y pago de matrícula; el 76\%, no trabajaba. Carvajal Olaya et al. (2006) y Restrepo (2010) reportan que el $76 \%$ de los estudiantes desertores no trabajaban durante el tiempo que estuvieron vinculados a la Universidad y el $28 \%$, sí lo hacía.

La condición de ser trabajadores durante la época de estudios no es una causa exclusiva de la deserción, pero influye, como se menciona en la investigación del Ministerio de Edu- 
cación y Centro de Estudios sobre el Desarrollo Económico (MEN, 2008), si se tiene en cuenta que los ingresos económicos familiares afectan o inciden en la decisión de desertar. El estudio muestra que los problemas de escasos o mínimos recursos, también se trasladan a la educación superior y obligan a los estudiantes a abandonar el sistema educativo, en búsqueda de ingresos adicionales para el hogar.

Lo referido por los desertores, en cuanto a la orientación vocacional, el $67,8 \%$ la recibió, mientras que el 32,2\%, no tuvo acceso a ella. A pesar que la mayoría de los estudiantes quienes desertaron recibió orientación vocacional, la razón de deserción está articulada con la situación económica y académica. Es importante tener en cuenta que en Colombia, los menores niveles de deserción se dan en las programas de ciencias de la salud y se relaciona con factores disciplinares, pedagógicos y vocacionales importantes, que entran en juego durante el trayecto académico (MEN, 2010); no obstante, para los programas de Enfermería es un fenómeno real y, sobre el cual, se debe reflexionar, en cuanto al número de candidatos que cumplen con el perfil, los mecanismos de selección para disminuir el riesgo de deserción, cómo influyen los procesos académicos en la decisión de desertar y el acompañamiento a los estudiante, durante su proceso de formación.

Las causas de deserción para Enfermería están asociadas con factores económicos, en un $28,8 \%$; académicos, $22 \%$; la unión de los factores económicos y académicos, con un $15 \%$; la falta de orientación vocacional, 11,9\%; las situaciones de salud, como enfermedad o el embarazo, corresponden al 6,8\% y el no adaptarse al ambiente universitario, un $7,1 \%$; también se registran otros factores, como el cambio de lugar de residencia a otra ciudad, con un 5\%; los familiares, el 1,7\% y la unión de tres factores, como los económicos, académicos y familiares, presenta el 1,7\%. Múltiples factores coadyuvan en la decisión de desertar de los programas.

Las investigaciones revisadas en diferentes instituciones presentan las causas de deserción en orden de prioridad y cambian de acuerdo a la metodología utilizada, por lo tanto, es difícil comparar los resultados de deserción entre las instituciones, por las particularidades de las mismas.

Para la Universidad de San Buenaventura, el 65\% de los participantes abandonó su formación profesional por diversas razones, como: problemas de salud, traslado de país, inconformidad con el pensum, vivir fuera de Bogotá, inseguridad de asumir la carrera, incompatibilidad del horario de estudio con el horario de trabajo y embarazo. El 35\%, por dificultades económicas, que motivaron el retiro de la Universidad (Mateus Rodríguez et al. 2011).

El factor académico, para los estudiantes de la U.D.C.A, ocupan el segundo lugar, con un $22 \%$, mientras que en la Uni- versidad de los Llanos es la principal causa, con un $47,8 \%$ (Malagón Escobar et al. 2007). En la Universidad del Cauca esta causa refleja una diferencia notoria en la deserción; se muestra el factor principal bajo rendimiento, discriminado por género, 66,7\%, en mujeres y, en hombres, 33\% (Chávez et al. 2002). En relación con el promedio académico de los desertores, el 39\% de las mujeres obtuvo un promedio en el rango de 3,1 a 4,0; el 33\%, de 2,1 a 3,0; el 15,7\%, de 0,0 a $1,0 \mathrm{y}$, el $2 \%$, entre 1,1 a 2,0. Para los hombres desertores, el $37 \%$ obtuvo un promedio académico, en el rango de 2,1 a 3.0; el 25\%, promedios entre 1,1 a 2,0 y, el $12 \%$, un promedio académico entre de 0,0 a 1,0. Las mujeres tenían un promedio académico más alto, comparativamente con los hombres, lo que refleja que la situación académica puede ser recuperable y que las causas que determinaron la salida están relacionadas con otros factores, como las económicas, las personales o las socio-culturales.

Existe evidencia que los varones son más propensos a renunciar a los programas de Enfermería y Partería (Stott, 2004), probablemente, asociado al predominio femenino de la profesión.

En cuanto al número de periodos académicos cursados por los estudiantes quienes abandonaron el programa, se identificó que el 50,9\%, cursó un periodo académico; el 22,8\%, dos periodos; el $17,5 \%$, tres; el $7 \%$ cursó cinco periodos académicos y, el 1,8\%, cuatro periodos. Los datos coinciden con los presentados en la Universidad Libre de Pereira, con el programa de Enfermería, en donde la deserción ocurrió durante los primeros semestres de la carrera, siendo la mayor proporción en primero, con $27,8 \%$ y, segundo semestre académico, con 16,7\%; el 2,8\%, en el último (Restrepo, 2010).

El MEN y el Centro de Estudios sobre Desarrollo Económico (CEDE) de la Universidad de Los Andes, en un estudio de seguimiento, con participación de 70 instituciones de educación superior, en el periodo 1998-2005, encontró que la tasa de deserción más alta, con un $23,5 \%$, ocurrió en el paso entre el primero y el segundo semestre, lo cual, equivale a que, aproximadamente, uno de cada cuatro estudiantes abandona sus estudios al iniciar el primer año de su programa académico. Al finalizar el cuarto semestre, la tasa de deserción acumulada fue de $39 \%$, es decir, del $100 \%$ de los estudiantes que iniciaron, solamente el $61 \%$ continuaba sus estudios en el quinto semestre (MEN, 2008). Los estudios de deserción muestran que en la medida que el estudiante avanza en su proceso de formación disminuye la probabilidad de abandonar el programa, de mejorar el rendimiento académico, de adaptarse y vincularse a los procesos de acompañamiento y a la vida universitaria. En estudio realizado en Costa Rica sobre deserción universitaria con relación al momento en que ocurre la deserción, el mayor número se sitúa en el primer año de estancia universitaria (Abarca Rodríguez E Sánchez Vindas, 2005), comportamiento similar para Colombia. 
A la pregunta de si busco ayuda antes de desertar, la mayoría de los estudiantes de Enfermería, es decir, el 54,2\%, respondió que no; el 45,8\%, sí busca ayuda; el 15,3\%, acudió a sus padres y el 19,2\%, solicitó ayuda a dos o más personas. Esta situación es un indicador del apoyo que requieren los estudiantes con riesgo de deserción, de los programas de retención, apoyo de tutores que los acompañen y los orienten para buscar ayuda oportunamente.

Los resultados frente al interés de determinar la actividad luego de desertar del Programa de Enfermería en la U.D.C.A, identificó que el 37,3\% realizó estudios en otros programas académicos, de carácter administrativo, como Contaduría, Ingeniería de Sistemas y Administración de Empresas, mientras que el 62,7\%, no realizó estudios posteriores, dedicándose a actividades laborales y del hogar; el 8,5\% continuó desarrollando actividades académicas en el programa académico de Enfermería en otra institución. En el estudio realizado en la Universidad de Ibagué, Rojas Betancur (2008) refiere, como resultado de la deserción, el 50,8\%, se dedica a trabajar; el $42,4 \%$, a estudiar; el $13,6 \%$, estudian y trabaja simultáneamente y el $6,8 \%$, se dedican a otras ocupaciones, como el hogar.

La mayoría de los estudiantes, quienes abandonan los estudios, se vinculan laboralmente; si han recibido apoyo de sus familias buscan obtener ingresos económicos, que les permite apoyar a la familia o para su propio sostenimiento. Es claro que no abandonan su interés por estudiar y persiguen nuevas oportunidades, para iniciar su formación. Pero reiniciar los procesos tiene implicaciones, como aumentar costos económicos y asumir el riesgo de abandonar, definitivamente, la idea de formarse. El gobierno y las instituciones de educación deben preocupar por mantener programas y sistemas de apoyo, que promuevan la permanencia de los estudiantes.

Los resultados obtenidos con relación a factores asociadas a la deserción corresponden con los revisados en la literatura; los económicos son la principal causa, seguidos de los factores académicos, que llevan a los estudiantes a abandonar sus estudios, antes de alcanzar el título.

El mayor porcentaje de deserción se da en los primeros semestres, especialmente, en el primer periodo académico; el número de desertores, se relaciona con el número de estudiantes matriculados: a mayor número de estudiantes matriculados mayor deserción. La mayoría de desertores corresponde a estudiantes solteros, de género femenino y con edades de 16 a 20 años. Los factores socio-culturales son otras variables que se tienen en cuenta, como el no adaptarse al ambiente universitario, el cambio de lugar de residencia a otra ciudad, la dedicación a los estudios y situaciones familiares, que generan deserción en una mínima proporción, según lo referido por los estudiantes.
Las Universidades deben evaluar el impacto que genera la deserción, desde el esfuerzo para los profesores que dedican trabajo y tiempo al acompañamiento y si no se logra culminar, representa una pérdida para la institución y para la profesión de potenciales enfermeras, quienes no tuvieron la oportunidad de recibir apoyo, para continuar su proceso de formación.

El estudio de deserción muestra que los aspectos económicos tienen un gran peso, por lo tanto, el gobierno y las Universidades se deben preocupar por buscar estrategias de financiación, que garanticen el ingreso y la permanencia de los estudiantes, en las Instituciones de Educación Superior.

Como recomendación es fundamental mantener políticas de retención estudiantil, que permita identificar a los estudiantes con mayor probabilidad de deserción y hacer seguimiento durante los cuatro primero periodos académicos. Emplear estrategias para identificar potenciales factores de riesgo, como bajo rendimiento académico, adaptación al ambiente universitario y dificultades económicas.

Conflicto de intereses: El manuscrito fue preparado y revisado con la participación de todos los autores, quienes declaramos que no existe ningún conflicto de intereses que ponga en riesgo la validez de los resultados presentados. Financiación: Este estudio fue financiado por la Universidad de Ciencias Aplicadas y Ambientales U.D.C.A, en la modalidad de joven investigador.

\section{BIBLIOGRAFÍA}

1. ABARCA RODRÍGUEZ, A.; SÁNCHEZ VINDAS, M.A. 2005. La deserción estudiantil en la educación superior: el caso de la Universidad de Costa Rica. Rev. Electrónica Act. Invest. Educ., vol. 5. Disponible desde Internet en:http://redalyc.uaemex.mx/ pdf/447/44759911.pdf (con acceso 09/12/2012).

2. BOWDEN, J. 2008. Why do nursing students who consider leaving stay on their courses? Nurse Res. (London). 15(3):45-58.

3. BRAVO CASTILLO, M.; MEJÍA GIRALDO, A. 2010. Los retos de la educación superior en Colombia: una reflexión sobre el fenómeno de la deserción universitaria. Rev. Ed. Ing. (Colombia).1(10):85-98.

4. BRAXTON, J.M.; SHAW-SULLIVAN, A.; JOHNSON, R.M. 1997.Appraising Tinto's Theory of college students departure. En: Smart, J.C. (ed). Higher Education Handbook of Theory and Research. (12). Agathon Press. (United States). p.359-384. 
5. CAMERON, J.; ROXBURGH, M.; TAYLOR, J.; LAUDER, W. 2011. An integrative literature review of student retention in programs of nursing and midwifery education: why do students stay? J. Clinic. Nurs. (España). 20(9/10):1372-1382.

6. CARVAJAL OLAYA, P.; TREJOS CARPINTERO, A.A.; MEJÍA CORDOBES, J. 2006. Deserción estudiantil, Facultad de Ciencias de la Salud 2000 - 2004. Análisis de correspondencias múltiples. Rev. Médica de Risaralda. 12(2):30-42.

7. CHÁVEZ G., M.; ORTIZ DE COLLAZOS, O.; TORRES, C.I. 2002. Deserción estudiantil en Enfermería: "una mirada holística y una propuesta de intervención". Universidad del Cauca. 72p.

8. DÍAZ PERALTA, C. 2008. Modelo conceptual para la deserción estudiantil universitaria chilena. Rev. Est. Pedag. (Chile). 34(2):65-86.

9. DURKHEIM, E. 1951. Suicide. Ed. Free Press (New York). 450p.

10. FISHBEIN, M.; AJZEN, I. 1975. Attitudes toward objects as predictors of simple and multiple behavioral: An introduction to theory and Research Ed. AddisonWesley, Reading, MA. (EEUU). 573p.

11. GONZÁLEZ F, L.E. 2005. Repitencia y deserción en América Latina. Informe Sobre la Educación Superior en América Latina y el Caribe. Unesco lesalc boletin@unesco.org.ve (Chile): 156-168. Disponible desde Internet en: http://www.iesalc. unesco.org.ve/dmdocuments/biblioteca/libros/ InformeES-2000-2005.pdf (con acceso 20/06/2012).

12. GLOGOWSKA, M.; YOUNG, P.; LOCKYER, L. 2007. Should I go or should I stay? A study of factors influencing students' decisions on early leaving. Act. Learn. Higher Educ. (UK). 8(1):63-77.

13. HIMMEL, E. 2002. Modelos de análisis de la deserción estudiantil en la educación superior. Retención y movilidad estudiantil. Rev. Calidad Edu. Consejo Nal. Ed. (Chile). 17(2):91-108.

14. JEFFREYS, M.R. 2010. Nursing student. Retent understanding the process and making a difference. Ed. Springer Publ. Co. (N. Y.) 351p.

15. JEFFREYS, M.R. 2007. Tracking students through program entry, progression, graduation and licensure: assessing undergraduate nursing student retention and success. Nurse Educ. Today. (UK). 27:406-419.

16. NIGENDA, G.; RUIZ, J.A.; ROSALES, Y.; BEJARANO, R. 2006. Enfermeras con licenciatura en México: estimación de los niveles de deserción escolar y desperdicio laboral. Rev. Salud Públ. Méx. 48(1):22-29.

17. MALAGÓN ESCOBAR, L.M.; SOTO HERNÁNDEZ, E.L.; ESLAVA MOCHA, P.R. 2007. Deserción la universidad de los Llanos (1998-2004). Rev. Orinoquia. (Colombia). 11(1):23-40.

18. MATEUS RODRÍGUEZ, M.J.; HERRERA HERNÁNDEZ, C.; PERILLA SUÁREZ, C.; PARRA QUECÁN, G.; VERA MALDONADO, A. 2011. Factores presentes en la deserción universitaria en la Facultad de Psicología de la Universidad de San Buenaventura, sede Bogotá en el periodo comprendido entre 19982009. Rev. Psychol.: Avances Disciplina. (Colombia). 5(1):121-133.

19. MINISTERIO DE EDUCACIÓN NACIONAL (MEN). 2010. Educación superior ingreso, permanencia y graduación. p.1-20. Disponible desde Internet en: http://www.mineducacion.gov.co/ sistemasdeinformacion/1735/articles-254702 boletin_14.pdf (con acceso 15/08/2012).

20. MINISTERIO DE EDUCACIÓN NACIONAL (MEN). 2009. Deserción estudiantil en la educación superior Colombiana. Metodología de seguimiento diagnóstico y elementos para su prevención. 158p. Disponible desde Internet en: http://www.mineducacion.gov.co/ sistemasdeinformacion/1735/articles-254702_libro_ desercion.pdf (con acceso 15/08/2012).

21. MINISTERIO DE EDUCACIÓN NACIONAL (MEN). 2008. Análisis de determinantes de la deserción en la Educación Superior Colombiana. Con Base en SPADIES. 96p Disponible desde Internet en: http://www. mineducacion.gov.co/sistemasdeinformacion/1735/ articles-254702_libro_desercion.pdf(con acceso 15/ $08 / 2012$ ).

22. PRYJMACHUK, S.; EASTON, K.; LITTLEWOOD, A. 2009. Nurse education: factors associated with attrition. J. Adv. Nurs. (UK). 65(1):149-160.

23. RESTREPO V, A. 2010. Factores relacionados con la deserción estudiantil en el programa de enfermería de la Universidad Libre de Pereira. Cultura del cuidado enfermería. (Colombia). 7(2):1-10.

24. ROJAS BETANCUR, M.; GONZÁLEZ, D.C. 2008. Deser- 
ción estudiantil en la Universidad de Ibagué, Colombia: una lectura histórica en perspectiva cuantitativa. Zona próxima 1(9):70-83. Disponible desde Internet en: http://redalyc.uaemex.mx/src/inicio/ArtPdfRed. jsp?iCve $=85312286006$. ISSN 1657-2416 (con acceso 27/03/2011).

25. ROJAS BETANCUR, M. 2008. La deserción estudiantil en la Universidad de Ibagué: la perspectiva de los "desertores". Rev. Virtual U. Católica del Norte (Colombia). 25(1):1-17.

26. SALDAÑA, V.; BARRIGA, O. 2010. Adaptación del modelo de deserción universitaria de Tinto a la universidad Católica de la Santísima Concepción, Chile. Rev. Cienc. Soc. (Colombia) 16(4):616-628.

27. SANABRIA, H. 2002. Deserción en estudiantes de enfermería en cuatro universidades del Perú. Anal. Fac. Med. (Peru). 63(4):301-311.

\section{SISTEMA PARA LA PREVENCIÓN DE LA DESERCIÓN} DE LA EDUCACIÓN SUPERIOR (SPADIES). 2005. Disponible desde Internet en:http://www. mineducacion.gov.co/sistemasdeinformacion/1735/ w3-article-212299.html (con acceso 30/04/2012).

29. SPADY, W. 1970. Dropouts from higher education: An interdisciplinary review and synthesis. Interchange. 19(1):109-121.
30. STOTT, A. 2004. Issues in the socialization process of the male student nurse: implications for retention in undergraduate nursing courses. Nurse Educ. Today. (UK). 24 (2):91-97.

31. TINTO, V. 1989. Definir la deserción: una cuestión de perspectiva. Rev. Ed. Superior (México). 71:33-51.

32. UNESCO/IESALC Organización de la Naciones Unidas para la Educación la Ciencias y la Cultura /Instituto Internacional Para La Educación Superior en América Latina y el Caribe. 2006. Informe sobre la Educación Superior en América Latina y el Caribe 20002005 Talca Repitencia y deserción universitaria en América Latina. p.156-168.

33. YEPES, F.L.; BELTRAN SALAZAR, M.; ARRUBLA, J.; MARIN, L.M.; MARTÍNEZ, M.; TOBON, C.; HOYOS, A.M. 2007. Factores causales de la deserción estudiantil en el pregrado de la Facultad de Odontología de la Universidad de Antioquia de 1997 a 2004. Rev. Fac. Odontol. (Colombia). 19(1):35-48.

Recibido: Noviembre 8 de 2012

Aceptado: Septiembre 3 de 2013

Como citar:

Piratoba H., B.N.; Barbosa Ch., O.O. 2013. Factores de deserción de los estudiantes en la facultad de enfermería de la Universidad de Ciencias Aplicadas y Ambientales U.D.C.A, durante el periodo: 2009-2010-I 2011. Rev. U.D.C.A Act. \& Div. Cient. 16(2): 553-562. 